\title{
Peertechz
}
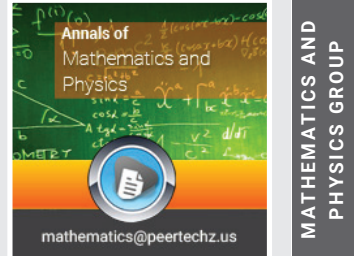

Research Article

\section{Drag force through gases and plasma}

\section{Apostol*}

Department of Theoretical Physics, Institute of Atomic Physics, Magurele-Bucharest Mg-6, POBox MG-35, Romania
Received: 30 December, 2021

Accepted: 24 January, 2022

Published: 25 January, 2022

*Corresponding authors: M Apostol, Professor, Department of Theoretical Physics, Institute of Atomic Physics, Magurele-Bucharest Mg-6, POBox MG-35,

Romania, Tel: 0727438 755;

E-mail: apoma@theory.nipne.ro

Copyright License: (c) $2022 \mathrm{M}$ Apostol. This is an open-access article distributed under the terms of the Creative Commons Attribution License, which permits unrestricted use, distribution, and reproduction in any medium, provided the original author and source are credited.

https://www.peertechzpublications.com

Check for updates

\section{Abstract}

The drag force in a gas (previously derived by Stokes and Rayleigh) is derived by means of the molecular kinetics (transport equation of the momentum). Two regimes of resistance to motion are identified, governed by the relation of the velocity to the thermal (molecular) velocity. They correspond to the molecular movement, for small velocities, or to the hydrodynamic motion for high velocities. In the former case sound waves are not excited, and energy is dissipated by viscosity (friction), while in the latter case the energy is dissipated by the excitation of the sound waves. Also, the treatment is applied to the plasma. It is shown that in usual plasmas it is unlikely that the body motion excites plasmons.

The drag force is well known in fluid mechanics [1-5]. For small velocities (small Reynolds numbers) it is proportional to the velocity of the body moving through the fluid (Stokes force), while for larger velocities (higher Reynolds numbers) it is proportional to the squared velocity (Rayleigh force). In the former case, the energy is dissipated by viscosity, while sound waves (including shock waves) are excited in the latter case. In potential flow, the fluid mechanics predicts no drag (d'Alembert paradox). In viscous fluids, the Stokes law implies the boundary-layer theory. We give in this Note a derivation of the drag force by means of molecular kinetics, which provides a unitary treatment and avoids the difficulties of the fluid mechanics (d'Alembert paradox included).

Let us consider a plane solid surface (solid body) placed vertically in the $y, z$-plane and moving horizontally with velocity $v>0$ along the $x$-axis through an ideal classical gas. The energy and momentum conservation laws for an elastic collision of the surface with a gas molecule are

$$
\begin{gathered}
M v^{2}+m v_{x}^{2}=M v^{2}+m v_{x}^{2}, \\
M v+m v_{x}=M v^{\prime}+m v_{x}^{\prime},
\end{gathered}
$$

where $M$ is the mass of the body, $m$ is the molecular mass, $v^{\prime}$ is the velocity of the body after the collision, and $v_{x}, v_{x}^{\prime}$ are the molecule velocities before and after the collision, respectively. We assume that the collisions along the $y, z$-directions are balanced, such that they do not change the states of motion. Also, we note that the velocities in equations (1) are algebraic (with their sign). From equations (1) we get

$$
\begin{gathered}
v^{\prime}=\frac{1-m / M}{1+m / M} v+\frac{2 m / M}{1+m / M} v_{x}, \\
v_{x}^{\prime}=\frac{2}{1+m / M} v-\frac{1-m / M}{1+m / M} v_{x} .
\end{gathered}
$$

The momentum $(\Delta P)$ and energy $(\Delta E)$ changes for the body are

$$
\begin{gathered}
\Delta P=M\left(v^{\prime}-v\right)=-\frac{2 m}{1+m / M}\left(v-v_{x}\right), \\
\Delta E=\frac{1}{2} M\left(v^{2}-v^{2}\right)=-\frac{2 m\left(v-v_{x}\right)}{(1+m / M)^{2}}\left(v+\frac{m}{M} v_{x}\right) .
\end{gathered}
$$


Let us consider now those molecules with velocity $v_{\mathrm{x}}<0$ along the $x$-axis. These molecules collide with the body, on the right side $(x>0)$. The number of collisions per unit time and the unit area is $n\left(v-v_{x}\right)$, where $n$ is the gas density. It follows that a force per unit area $p_{1}=\Delta P \cdot n\left(v-v_{x}\right)=-\frac{2 m n}{1+m / M}\left(v-v_{x}\right)^{2}, v_{x}<0$

occurs on the right side of the body; it is directed along with the negative $x$-axis. If we average this force over all velocities $v_{x}<0$, we get the corresponding pressure. Let us consider now the molecules with velocity $v_{x}>0$. For $v_{x}>v$, they collide with the body on the left side; the number of collisions per unit time and the unit area is $n\left(v_{x}-v\right)$. If $v_{x}<v$, the body collides with these molecules on the right side; the number of these collisions per unit time and the unit area is $n\left(v+v_{x}\right)$. It follows that we get the pressure

$$
\begin{gathered}
p_{2}=\frac{2 m n}{1+m / M}\left(v_{x}-v\right)^{2}, v_{x}>v, \\
p_{2}=-\frac{2 m n}{1+m / M}\left(v^{2}-v_{x}^{2}\right), 0<v_{x}<v,
\end{gathered}
$$

where the average must be taken over the values of $v_{x}$ which are indicated in each row of equations (5). These averages imply truncated integrals of Gaussians. It is convenient to estimate the pressure in two distinct cases: $v<\left\langle v_{\text {th }}\right.$ and $v>>v_{\text {th }}$, where we take for the thermal velocity $v_{t h}=(T / 2 \pi m)^{1 / 2}, T$ being the temperature. For $v<<v_{\text {th }}$ we may neglect the second raw in equations (5), such that the total pressure is given by adding the first row of equations (5) and equation (4). We get

$$
p \simeq-\frac{8 m n}{1+m / M}-\bar{v} x, v_{x}>0, v \ll v_{t h} .
$$

In the second case, $v>>v_{\text {th }}$, we may neglect the first row in equations (5) and add the second row and equation (4) to get the total pressure

$$
p \simeq-\frac{4 m n}{1+m / M}\left(\overline{v^{2}}+\bar{v} v_{x}\right) \simeq-\frac{4 m n}{1+m / M} \overline{v^{2}}, v_{x}>0, v \gg v_{t h} .
$$

The final result is

$$
\begin{aligned}
& p \simeq-\frac{8 m n}{1+m / M} v v_{t h}, v \ll v_{t h}, \\
& p \simeq-\frac{2 m n}{1+m / M} v^{2}, v \gg v_{t h} .
\end{aligned}
$$

We can see that in both cases a drag force occurs (opposite to the direction of motion). An interpolation formula is

$$
p \simeq-\frac{2 m n}{1+m / M}\left(v^{2}+4 v v_{t h}\right) .
$$

We may neglect the ratio $m / M$ in the denominator of equation (9) and replace $m n$ it with the mass density $\rho$; for a macroscopic body the force implies the area $A$ of its crosssection, such that, for small velocities the drag force is

$$
F \simeq-8 \rho v_{t h} A v
$$

for a small body with dimension $r$ the effective area is reduced by transverse collisions to $\delta A=r_{\wedge}$, where $\wedge$ is the mean free path and the force becomes $F \simeq-8 \rho \Lambda v_{t h} r v$; the product $\rho \wedge v_{\text {th }}$ is the viscosity $\eta$, such that the force is $\simeq-8 r \eta v$, which is approximately Stokes' formula [2] $-6 \pi r \eta v$ for a sphere of radius $r$. For high velocities, the formula was derived by Rayleigh by means of the fluid dynamics [7]. It is worth noting that the pressure $p \simeq-2 \rho v^{2}$ given by equation (8) $v>>v_{\text {th }}$ is four times larger than the hydrodynamic pressure $\frac{1}{2} \rho v^{2}$.

The drag force is derived here by using molecular kinetics (equivalent to transport equation with a macroscopic velocity $v$ ); the transported quantity is momentum. If we leave aside the molecular velocities, as for microscopic, but large, amounts of fluid, we may get Euler's equation of motion of fluids (NavierStokes equations). This was the original approach of Stokes (who used the empirical viscosity) and Rayleigh. Further on, for low velocities, from Euler's equation, we may get the NavierCauchy equations of elastic motion. For low velocities these equations predict sound waves, in particular the hydrodynamic sound, with velocity $c=\sqrt{c_{p} T / c_{v} m}$ in an ideal classical gas, where $c_{\mathrm{p}, \mathrm{v}}$ are specific heats at constant pressure and volume, respectively. We note that $c$ is close to the molecular thermal velocity $\simeq \sqrt{T / m}$. Sound is a collective excitation, which implies local thermal equilibrium, but not a global equilibrium: the pressure, the density, and the temperature vary on a global scale (it is a mechanical motion compatible with the statistical motion; the sound is not a transport phenomenon).

Let us examine first the case $v<<v_{\text {th }}$. Let us assume a local displacement $u$ in the gas; we assume that it is sufficiently small to produce a small pressure imbalance $\delta p$; since $u<<\Lambda$ this imbalance is adiabatic, i.e. the molecules do have not enough time to collide with each other. This displacement produces a small density imbalance $\delta \rho=-\rho \operatorname{divu}$. According to Euler's equation $\ddot{\mathbf{u}}=-\frac{1}{\rho} \operatorname{grad} \delta p$, we get

$$
\frac{\partial^{2} \mathbf{u}}{\partial t^{2}}=-\frac{1}{\rho}\left(\frac{\partial p}{\partial \rho}\right)_{S}(-\rho g r a d \operatorname{div} \mathbf{u})=\left(\frac{\partial p}{\partial \rho}\right)_{S} \operatorname{grad} \operatorname{div} \mathbf{u},
$$

which is the equation of sound propagating with velocity given by $c^{2}=(\partial p / \partial \rho)_{S}$, where the suffix $S$ means constant entropy (S). Since $(\partial p / \partial \rho)_{S}==\left(c_{p} / c_{v}\right)(\partial p / \partial \rho)_{T}$, we get the sound velocity given above (where). This is the hydrodynamic 
sound in an ideal gas. Since it is collisionless, it follows that it cannot be excited by the motion of the body through the gas, as long as $v<<v_{\text {th }}$. In particular, the density variations produced by the sound are $\delta p=c^{2} \delta \rho=-\rho c^{2} d i v \mathbf{u}=-\frac{\rho c p^{T}}{c_{v} m} d i v \mathbf{u}$, while the pressure variation given by equations ( 8$)$ is $\delta p=-8 \rho \sqrt{\frac{T}{2 \pi m} v}$.

This latter pressure does not produce variations of the density. The sound is a local-equilibrium phenomenon, while the drag force is caused by friction (viscosity), which, on its short scale, is a non-equilibrium phenomenon. The drag force dissipates energy, by mechanical work, which, per unit time, can be computed from the lost energy given by equations (3).

The situation is different in the regime $v>>v_{t h}$; in this case, the body generates variations of the density, and, therefore, excites sound waves. It is worth noting that the pressure $-2 \rho v^{2}$ given by equations (8) in this case does not depend on $v_{t h}$, as if the molecular movement would be immaterial. This is the hydrodynamic regime. Indeed, let us assume that $V h(r)$ is the characteristic function of the body, where $V$ is the volume of the body; the characteristic function of the gas is $f(r)=1-V h(r)$. For a pointlike body, we can take $h(r)=\delta(r)$. We are interested in the variation $\delta f$ of this function due to the movement of the body, i.e. the variation of the function $f(r-v t)=1-V h(r-v t)$, where $t$ denotes the time. The variation $\delta F$ of any quantity $F$ associated with the body is given by $\delta F=F \delta f$. The variation of this function can be written as $\delta f=V \mathbf{v} g r a d h(\mathbf{r}-\mathbf{v} t) \delta t$. As long as $v<<v_{\text {th }}$ the time $\delta t$ is much longer than the mean collision time $T$, such that the equilibrium is restored rapidly, and the variation $\delta f$ is zero. If $v>>v_{\text {th }}$ the time $\delta t$ is much shorter $T$, we have an estimate $\delta f=V \tau \mathbf{v} g r a d h(\mathbf{r}-\mathbf{v} t)$ for the variation $\delta f$. Therefore, we have a variation of the pressure $\delta p=-\left(2 \rho v^{2}\right) V \tau \mathbf{v} \operatorname{gradh}(\mathbf{r}-\mathbf{v} t)$ and a variation of the density

$$
\delta \rho=\rho V \tau \mathbf{v} \operatorname{gradh}(\mathbf{r}-\mathbf{v} t)=\rho V \tau \operatorname{div}[\mathbf{v} h(\mathbf{r}-\mathbf{v} t)] .
$$

Since $\delta \rho=-\rho d i v \mathbf{u}$, where $\mathrm{u}$ is the displacement associated with the density variations, we get the displacement

$$
\mathbf{u}=-V \tau \mathbf{v} h(\mathbf{r}-\mathbf{v} t)
$$

obviously, this displacement satisfies the equation

$$
\frac{\partial^{2} \mathbf{u}}{\partial t^{2}}-(\mathbf{v g r a d})^{2} \mathbf{u}=0
$$

or

$$
\frac{\partial^{2} u}{\partial t^{2}}-v^{2} \frac{\partial^{2}}{\partial r^{2}} u=0
$$

where $\mathrm{u}$ and $\mathrm{r}$ are directed along with the velocity $\mathrm{v}$ (and $\mathrm{u}$ is restricted to the cross-section of the body); this is a shock wave. Due to the equilibrium movement, the thickness of the tail increases in time with velocity $\mathrm{v}_{\mathrm{th}} / \mathrm{u}$. The displacement $\mathrm{u}$ can be expanded in a Fourier series

$$
\mathbf{u}=-V \tau \mathbf{v} \frac{1}{(2 \pi)^{3}} \int d \mathbf{k} h(\mathbf{k}) e^{i(\mathbf{k r}-\mathbf{k v} t)},
$$

which shows that the disturbance is a superposition of sound waves with frequency $w=v k=\mathrm{c}(\mathrm{v} / \mathrm{c}) k$; since the wavevector is localized over a range $\Delta k \simeq 1 / l$, where $l$ is the dimension of the body, it follows that the sound extends up to a frequency of the order $\Delta \omega \simeq v / l$. The movement of the body in this case dissipates energy by exciting sound waves. It is worth noting that equations (14) and (15) are hydrodynamic equations, where there is no external force: the force exerted by the body on the gas is taken by the molecular movement, which determines (through $T$ ) the amplitude of the free sound waves.

The above treatment can also be used for plasma. Let us assume an ionized gas, and consider only its ionized component. The density of the gas and the density of the plasma are very low. The electrons $(e)$ have a temperature $T_{e}$, while the ions (i) have a temperature $T_{\mathrm{i}}$, such that $T_{\mathrm{e}}>>T_{\mathrm{i}}$. Both temperatures are much higher than the Coulomb energy $\mathrm{q}^{2} / a$, where $q$ is the electron (ion) charge and $a$ is the mean separation distance between the particles. The electrons are correlated to the ions, through the Debye length, which in this case is very large. The corresponding cross-sections are $\sigma_{e-e}=\left(q^{2} / T_{e}\right)^{2}$ , $\sigma_{i-i}=\left(q^{2} / T_{i}\right)^{2}$ and $\sigma_{e-i}=q^{4} /\left(T_{e}^{2}+T_{i}^{2}\right)$; the mean freepaths are $\Lambda_{e-e}=\left(a^{2} / \sigma_{e-e}\right) a=a^{3} T_{e}^{2} / q^{4}, \Lambda_{i-i}=a^{3} T_{i}^{2} / q^{4}$ and $\Lambda_{e-i}=a^{3} T_{e}^{2} T_{i}^{2} / q^{4}\left(T_{e}^{2}+T_{i}^{2}\right) ;$ the mean lifetimes are $\tau_{e-e}=\Lambda_{e-e} / v_{e}=a^{3} T_{e} \sqrt{m T_{e}} / q^{4}, \tau_{i-i}=a^{3} T_{i} \sqrt{M T_{i}} / q^{4}$ and $\tau_{e-i}=a^{3} T_{e} T_{i} \sqrt{m M T_{e} T_{i}} / q^{4}\left(T_{e} \sqrt{m T_{e}}+T_{i} \sqrt{M T_{i}}\right)$, where $m$ is the electron mass and $m$ is the ion mass. If $T_{e} \sqrt{m T_{e}} \gg T_{i} \sqrt{M T_{i}}$ (which is the most realistic case), then $\tau_{e-i} \simeq \tau_{i-i} \ll \tau_{e-e}$, which shows that the electrons follow rapidly the ion motion, which ensures the equilibrium (electron-electron processes are immaterial for equilibrium); this is in accordance with the adiabatic hypothesis. In the unrealistic case, $T_{e} \sqrt{m T_{e}} \ll T_{i} \sqrt{M T_{i}}$ the electrons lag behind the ions. In this case there exist an ionic displacement $u_{i}$ and an electron displacement $u_{e}$, as well as a restoring polarization force which is responsible for a plasma frequency given by $\omega_{0}^{2}=4 \pi n q^{2} / \mu$, where $\mu$ is the reduced ion-electron mass. The ion-electron center of mass moves freely, with an ionic displacement coordinate which may be estimated as in equation (13); the electron component remains to be determined from boundary conditions. The 
ion-electron relative coordinate is subject to the polarization force and is a superposition of waves with frequencies given by $\omega^{2}=\omega_{0}^{2}+v^{2} k^{2}$. We note that this is a hydrodynamic regime, where the pressure force $\simeq-2 \rho v^{2}$, although hydrodynamic, is equilibrated by the molecular movement.

\section{Acknowledgment}

The author is indebted to the members of the Laboratory of Theoretical Physics at Magurele-Bucharest for many fruitful discussions. This work has been supported by the Scientific Research Agency of the Romanian Government through Grants 04-ELI / 2016 (Program 5/5.1/ELI-RO), PN 16420101 / 2016, PN 1909 01/2018, and PN (ELI) 16420105 / 2016.

\section{References}

1. Lamb H (1932) Hydrodynamics. Cambridge University Press, Cambridge, 1-8. Link: https://bit.ly/3rQnMRY

2. French AP (1970) Newtonian Mechanics, W. W. Norton\&Co., Inc., NY

3. Batchelor G (2000) An Introduction to Fluid Dynamics. Cambridge University Press, Cambridge. Link: https://bit.ly/3fSDAxV

4. Landau L, Lifshitz E (2004) Course of Theoretical Physics, Fluid Mechanics, Elsevier, Oxford 6. Link: https://bit.ly/3qYOgU3

5. Falkovich G (2011) Fluid Mechanics. Cambridge University Press, Cambridge Link: https://bit.ly/32uVUdF

6. Stokes GG (1851) On the effect of internal friction of fluids on the motion of pendulums. Trans. Cambridge Phil Soc 9. Link: https://bit.ly/3AsndSi

7. Strutt JW (1876) $3^{\text {rd }}$ Baron Rayleigh. On the resistance of fluids, Phil. Mag. II 430
Discover a bigger Impact and Visibility of your article publication with

Peertechz Publications

\section{Highlights}

* Signatory publisher of ORCID

* Signatory Publisher of DORA (San Francisco Declaration on Research Assessment)

* Articles archived in worlds' renowned service providers such as Portico, CNKI, AGRIS, TDNet, Base (Bielefeld University Library), CrossRef, Scilit, J-Gate etc.

* Journals indexed in ICMJE, SHERPA/ROMEO, Google Scholar etc.

* OAI-PMH (Open Archives Initiative Protocol for Metadata Harvesting)

* Dedicated Editorial Board for every journal

* Accurate and rapid peer-review process

* Increased citations of published articles through promotions

* Reduced timeline for article publication

Submit your articles and experience a new surge in publication services (https://www.peertechz.com/submission). 